\title{
VIDA Y SUBJETIVIDAD: LOS DESCARTES DE MICHEL HENRY
}

\section{Mario Lipsitz*}

\begin{abstract}
Michel Henry cede a Heidegger la palabra inicial de L'essence de la Manifestation (1964):
\end{abstract}

"Con el cogito sum, Descartes pretende ofrecer a la filosofía una base nueva y segura. Lo que, sin embargo, deja indeterminado en este comienzo "radical" es el modo de ser de la res cogitans, más precisamente, el sentido de ser del "sum"'"1.

Heidegger tiene razón: en su proyecto de sujetamiento universal Descartes ha olvidado por completo la cuestión decisiva del modo de ser de la substancia que es con certeza y que -ya en una perspectiva más fenomenológica que cartesiana- certifica al mundo ofreciéndole su ser y su sentido.

El olvido cartesiano ha sido a tal punto determinante que su rectificación justifica todo el proyecto filosófico de Henry y le confiere una significación positiva dentro de la problemática abierta por la enmienda del filósofo alemán al cogito cartesiano. En efecto, haciendo eco al exergo heideggeriano escribe inmediatamente Henry:

"El sentido del ser del ego es el tema de las presentes investigaciones.

Estas apuntan a elucidar, ante la mirada filosófica, aquello que entendemos cada vez que, a cualquier propósito y cada vez que es cuestión de nosotros mismos, decimos: Yo".

Lo vemos: Descartes, su proyecto, su método, sus resultados, no constituyen ciertos temas privilegiados en virtud de su peso histórico, encontrados por la marcha de un pensamiento original que necesariamente ha de medirse para hallar su propio lugar, sino, de algún modo, el comienzo

\footnotetext{
* Profesor de la Universidad Nacional de General Sarmiento (Buenos Aires). mlipstiz@ungs.edu.ar

${ }^{1}$ SZ, 24. Citado por M. Henry. L'essence de la manifestation, PUF, Paris, 1964, p. 1. En adelante (E.M).
} 
mismo de la filosofía de Michel Henry. Será también el lugar de un constante retorno.

Sin duda reside en esta habitualidad con los textos cartesianos y en la genial hermenéutica de Henry el que no haya un Descartes sino dos. Opuestos término a término. $\mathrm{O}$, más bien, como ha de quedar claro al final de este trabajo, un único y complejo Descartes donde el pensamiento se tensa y se relaja.

En efecto, el primer Descartes, sostenido en L'essence de la Manifestation y en la Phénoménologie du Corps (1965) entre las firmes pinzas de la conceptualidad fenomenológica para ser sometido a los filosos análisis de Henry, más interpretado que citado, nos invita a reconocer, por un lado, la genialidad del primer proyecto de fundación ontológica en la subjetividad y, por el otro, y por sobre todas las cosas, la absoluta impotencia de Descartes para llevarlo a cabo.

Se trata de la misma impotencia que, a la vez, Henry denuncia en la fenomenología y que se explicaría por los límites insuperables que, siguiendo a Descartes, se ha autoimpuesto a priori por su racionalismo $\mathrm{y}$, más esencialmente, por su intuicionismo.

El Descartes que se dibuja en la Genealogía del Psicoanálisis (1985) es muy otro. Tambien el proyecto de Henry es bien diferente: ya no se trata de hacer pie en una figura filosófica prácticamente construida como un concepto, para delinear a partir de sus supuestos, de su ambición y de su fracaso la posibilidad de otra filosofía ${ }^{2}$, sino de introducir un nuevo Descartes. En este libro de 1985, Descartes mismo pareciera tomar la palabra a través de -como solía decir Henry con cierto humor cuando se refería a sus propios emprendimientos hermenéuticos- "una nube de textos", polarizada por una tesis asombrosa. Una tesis que habría constituido el gran descubrimiento del mismo Descartes y que no es otra cosa que la primera formulación histórica de la tesis henryana: el Comienzo es absolutamente fenomenológico $y$ sin embargo no puede ser recogido en una intuición categorial, sensible o intelectual. La conciencia, la subjetividad, la Vida -tres nombres

${ }^{2}$ Precisemos que el interés de Henry por Descartes no es nunca el del erudito o el del historiador de la filosofía. En rigor, "ambos" Descartes intervienen en la obra de Henry como medios para señalar la posibilidad de "otra" filosofía: la de M. Henry. 
para el Comienzo- se autorrevela páticamente en una esfera de inmanencia previa al mundo e invisible a toda forma de ver, irreductible a toda "teoria".

Contra las interpretaciones más frecuentes, la problemática cartesiana del Comienzo no consistiría entonces en crear las condiciones para traer ante el ver de la intuición alguna evidencia irrecusable, sino en reconocer aquello que, reteniéndose fuera de la luz en una esfera de inmanencia absoluta, funda la posibilidad del ver de la intuición y de la evidencia en general: la subjetividad puramente pática del cogito.

Si en la historia de la filosofía, piensa Henry, Descartes fue el primero y uno de los pocos en haber comprendido que el verdadero Comienzo extiende su reino en una esfera de inmanencia esto le fue posible -contrariamente a lo sostenido por el Heidegger que abría L'essence de la Manifestationprecisamente por haber sabido problematizar el sentido de ser del ego cogitans y reconocer en este, como convenía, un modo de aparecer específico y beterogéneo respecto del modo de aparecer del mundo.

\section{Descartes I: La imposibilidad de pensar el fundamento}

Descartes es discutido por Henry desde el inicio de su primer gran libro L'essence de la manifestation (EM) en el contexto de una reflexión sobre los límites de una Ontología regional y de una Fenomenología racional. En esta discusión se pondrá de manifiesto la incapacidad de todo racionalismo a la hora de edificar una "Ontología Universal"3. El racionalismo cartesiano, en particular, realiza la posición del ego como Verdad, pero no sabe qué decirnos acerca del sentido de ser de ese ego sum, ni tampoco acerca de la enigmática singularidad de la conciencia sobre la que se apoya. Pero el racionalismo no es más que una de las realizaciones posibles del intuicionismo. Es finalmente el intuicionismo de Descartes quien impone un límite insuperable a su filosofía haciendole perder la posibilidad de pensar el fundamento.

3 "Ontología Fenomenológica Universal" es a nuestro entender la primera denominación de lo que sería la "Fenomenología Material" de M. Henry. Debe entenderse como una ontología de lo que Henry interpreta ya como Universal Concreto: el Ser. 


\section{El racionalismo y su impotencia para sostener una ontología universal}

La filosofía de Descartes intenta un verdadero "comienzo" y lo hace correctamente orientada por la intuición fenomenológica de confiarse a la cosa misma. Sin embargo, piensa Henry, ha olvidado por completo interrogarse acerca del contexto ontológico de la cuestión. Este olvido ha de condicionar negativamente el curso de la meditación cartesiana pues lo olvidado con él es, precisamente, el sentido del ser del sum.

En rigor, el ser del sum sólo constituye una región de ser particular y a este título, una investigación verdaderamente radical y conciente de sí debiera pretender superar esta limitación inadmisible para un Comienzo verdadero: en efecto, sólo una Ontologia Universal que supeditase la cuestión del ser del ego a la del sentido del ser en general podría estar en condiciones de dar respuesta adecuada al problema del sentido del ser del ego ${ }^{4}$.

El comienzo de 1'Essence de la Manifestation intenta, precisamente, elucidar la necesidad y también la posibilidad de subordinar la problemática sobre el Ego a una Ontología Universal. Con este fin, Henry interroga el lugar fundamental que la evidencia apodíctica ha ocupado en el cartesianismo y luego en la fenomenología como vía de acceso al ser del ego. Surge de este examen que la significación filosófica que ha recibido el cogito proviene exclusivamente del papel asignado a la evidencia y por ende, del racionalismo cartesiano. Henry señala entonces una curiosa consecuencia de este cogito racionalista: sólo a condición de no ser individual sino verdadera, la conciencia puede pretender ser fundamento para la ciencia exacta.

La paradoja extraordinaria que según Henry atraviesa a este cogito que busca apoyo en el ver de la ratio y en la evidencia es que la conciencia que buscaba lo universal y lo apodíctico ha terminado encontrándolo en la siempre impensada (por el racionalismo) singularidad de su propio ego.

La filosofía clásica ha intentado sortear esta paradoja que encubre, según Henry, una derrota, transformándola en una victoria: su simple estrategia ha consistido en afirmar que la conciencia no interviene en el cogito

${ }^{4}$ Digamos al pasar que aqui diverge radicalmente el proyecto henryano del proyecto del Heidegger de Ser y Tiempo: es absurdo, piensa Henry, interrogar a un ente particular (por ejemplo el Dasein) sobre el sentido del ser en general. 
a título de "individual" sino, precisamente, a titulo de "verdadera". Sin embargo, pregunta incisivamente Henry, si la evidencia no es otra cosa que la unidad de la posición racional con el dato que la motiva ¿qué otra cosa sino el dato intuitivo singular es lo que valida la posición de la conciencia?5

En efecto, "verdadera", la conciencia cartesiana sólo lo es en segunda instancia. en tanto se mantiene bajo la luz de la razón y de su propio tipo de evidencia, el único tipo de evidencia que realiza el ideal de perfección.

Es preciso entonces reconocer -concluye Henry- que el cogito ha surgido como primer elemento que realiza la apodicticidad que buscaba la ciencia racional, pero sobre el fondo de una unidad misteriosa y nunca pensada entre el ser verdadero y el ego particular sobre el que se apoya. La posición racional del ego como verdad no nos dice nada sobre la singular determinación en que hace pie y más bien nos oculta la indeterminación filosófica del sentido de ser de ese ego.

Ahora bien, ¿`se debiera confiar esta impostergable tarea de elucidación del ser del ego -incumplida por Descartes- a una fenomenología racional? Ante todo, responde Henry, debemos saber que la fenomenología tendría que inscribir modestamente esta tarea dentro del conjunto de investigaciones posibles que pretenden realizar una elucidación de las variadas estructuras del ser apuntando a sus caracteres eidéticos específicos ${ }^{6}$. En efecto, como nos lo ha mostrado Husserl, cada dominio del ser se presta a un tipo de posición racional y a una modalidad intuitiva determinada. Por este motivo es que, cuando lo que en última instancia está en la mira es una vista racional del ser en toda su amplitud, el ser del ego parece perder su privilegio.

El problema de fondo no es sin embargo el rebajamiento de dignidad del ego que tiene lugar cuando la elucidación racional del sentido de su ser es, digamos, "democráticamente" inscripta en el campo de ontologías regionales, sino que -considera Henry - toda tentativa de superación del ego cogito mediante una ampliación de la problemática que considere racionalmente el ser de todas las estructuras y regiones babria de resultar igualmente poco fecunda. En efecto, una investigación tal sobre el sentido del ser en general habría de seguir padeciendo del mismo lastre que la problemática del ego cogito pues

\footnotetext{
${ }^{5} \mathrm{EM}, 8$.

${ }^{6} \mathrm{EM}, 9$.
} 
"en tanto dicha investigación siga sometida al telos de la razón, seguirá apuntando únicamente al grado de validez y de legitimidad de las posiciones que opera la conciencia. Y su tarea infinita seguirá siendo una Idea, idea práctica y reguladora de un trabajo teórico infinito de orden ontológico. Cada vez que se trata de lograr la certeza respecto de algo, algo que es precisamente el correlato de esa certeza, y a ese titulo es el ser verdadero, (simplemente se obtiene) una verdad particular".

El proyecto cartesiano pretendía, con su método, garantizar al sujeto la evidencia del ser en su totalidad y resulta que, finalmente, muy por el contrario, en cada momento sólo nos entrega una evidencia particular sostenida bajo la luz por la intuición, es decir por el método, mientras que todo el resto de lo ente permanece en la incertidumbre, fuera del ser. Obviamente la crítica henryana del racionalismo, a través de Descartes apunta a la fenomenología intencional. Considerada en esta perspectiva original, la razón no aparece ya como la facultad de lo universal sino como un poder esencialmente atado a lo particular. Así, escribe Henry,

"obedeciendo al telos de la razón, en su pretensión de dominar los contenidos que ella es susceptible de legitimar, la filosofía se hunde progresivamente en el olvido de lo universal pues la razón no es una facultad de lo universal sino que por el contrario se dedica a descubrir verdades particulares cada vez que se consagra a determinar la validez de posiciones de conciencia en el cuadro de evidencia cuya estructura eidética está definida en su correlación con el sentido original del ser de una región dada".

La fenomenología racional, el racionalismo en general, condenarían a la filosofía a consagrarse a una ilimitada acumulación de verdades particulares. Al no permitir, por principio, superar el plano de la particularidad, no permiten superar tampoco el plano de lo puramente óntico. No permiten pues, la realización de una Ontología Universal susceptible de ofrecernos el sentido del ser en general.

Finalmente, dado que el sentido de ser de una región no puede ser aprehendido si el sentido del ser en general permanece indeterminado, el sentido del ser del sum ba de quedar en la oscuridad. El racionalismo de Descartes ha sido, pues, el primer motivo del fracaso de su proyecto de fundación ontológica en la subjetividad. De las manos del racionalismo, la tentación de lo particular, el olvido de lo Universal ha triunfado en el mismo momento 
en que Descartes, conducido por una legítima intuición filosófica se dirigía a la subjetividad viviente para interrogarla sobre el ser.

\section{El problema del intuicionismo cartesiano}

Sin embargo, lo que signa de entrada el fracaso del proyecto cartesiano -y desde luego el de la fenomenología, aun más que la absurda pretensión de que un sentido regional valga por universal, aun más que la extralimitación de la evidencia racional -típica del intelectualismo -que contradiciendo el tipo propio de evidencia de cada región pretende subsumir bajo su potestad la totalidad del campo de la experiencia intuitiva y también aun más que el extravío de la razón en lo particular es, según Henry, la esencial impotencia de la intuición en cuanto a la posibilidad de ofrecernos alguna realidad

En efecto, la intuición, que siempre está dirigida a una determinación particular es incapaz por principio de alcanzar el llenado perfecto que la teleología de la conciencia le prescribe, dado que cada dato intuitivamente dado conlleva un horizonte de datos significativos pero no perfectamente evidentes. Es en este horizonte imposible de ser totalmente reducido a la intuición que de alguna manera se encuentra oculto, sostiene Henry, el ser de la manifestación, esto es, su realidad.

Sin embargo, se podría objetar, ¿acaso el ego cogito no se ofrece con total certeza cuando en la intuición se toma a sí mismo por objeto? ¿No nos es dado entonces en su plena realidad?

El ego cogito es, efectivamente, un ente privilegiado que, tomándose a si mismo por objeto de intuición ciertamente cumple con el ideal de apodicticidad. ¿Cómo es esto posible? ¿No contradice por completo el análisis henryano de la intuición? $\mathrm{La}$ respuesta que ofrece el autor de l'Essence de la Manifestation a esta pregunta será decisiva, pues en ella se anuncia su propia filosofía así como la del Descartes no intuicionista que habrá de descubrir en los años 80 . Veámosla: el misterio del privilegio del ego cogito respecto de todos los otros entes, el misterio de su certeza, afirma Henry,

no reside en su naturaleza óntica u objetiva, no reside en su carácter de dato objetivo excepcional que supuestamente logra llenar por completo una intuición, sino justamente en su todavía impensada naturaleza ontológica. Es precisamente en aquello que en el ego cogito no puede 
ofrecerse como correlato de la intencionalidad de la conciencia, en aquello que no puede ser nunca dato intuitivo, que reside el origen y la realidad de la certeza con que se ofrece! $\mathrm{El}$ origen misterioso de la certeza y de la apodicticidad del ego cogito, sostiene Henry se encuentra fuera de la luz, en la naturaleza ontológica del ego: no en una supuesta capacidad excepcional de llenar enteramente una intencionalidad sino mas bien en lo contrario, en la imposibilidad de ofrecerse originariamente como dato exterior a una intuición y de cumplir por ella alguna forma de evidencia.

Lamentablemente, anota Henry, "la significación infinita de la identificación cartesiana de la certeza y la verdad" nunca ha sido comprendida correctamente. Ni siquiera por el mismo Descartes ${ }^{7}$.

Es preciso ahora afirmar que, en definitiva, el origen del olvido de lo Universal que se manifestaba como encantemiento por la determinación particular, como acumulación de verdades particulares y, en el plano metodológico, como incapacidad para construir una Ontología Universal se debe al intuicionismo de Descartes y no a su racionalismo. El racionalismo simplemente es el modo en que la indagación intuicionista encuentra, con la apodicticidad, su ideal de perfección.

La crítica henryana del intuicionismo tiene, desde luego, la intención de ir más allá de Descartes y de la fenomenología: es a la filosofía comprendida como teoría, como ver en la evidencia, que se dirige. En efecto, el papel decisivo que ha jugado el ego cogito en la filosofia se halla impregnado por un supuesto tan evidente como poco meditado: pues, observa Henry, es la filosofía misma, en tanto no es otra cosa que una modalidad de la vida intencional la que, obedeciendo a la idea de una "verdad" por alcanzar predetermina el lugar fundamental que ba ocupado la evidencia.

\section{La imposibilidad de pensar un cuerpo subjetivo}

Retomando los análisis de E.M, Michel Henry opone en su segundo gran libro, la Pbénoménologie du $\mathrm{Corps}^{8}$, un cogito biraniano al cogito cartesiano.

${ }^{7} \mathrm{EM}, 46$.

${ }^{8}$ Henry, Michel. Philosopbie et phenomenology du corps, PUF, Paris, 1965. En adelante $(\mathrm{PhC})$. 
Henry ha descubierto en Maine de Biran un verdadero dualismo del aparecer que, contrariamente al intuicionismo de Descartes, le ha permitido reconocer la esencia de la realidad fuera del horizonte ekstático donde opera la intuición. El sentimiento inmanente del esfuerzo, sostiene Henry, es en Biran sinónimo del conocimiento del yo.

Biran, a diferencia de Descartes no concibe

"que el ser del ego esté determinado como un puro pensamiento cuya esencia se agota en el conocimiento de la extensión y en la contemplación de las cosas sino que aparece ahora identificado con la acción por la que yo modifico continuamente el mundo, aunque más no fuera para hacer posible la continuación de mi propia existencia, con los movimientos que dirijo hacia el universo para alcanzarlo o huir de él. El ser del ego es el elemento mismo de estos movimientos. El ego es un poder, el cogito no significa un "yo pienso", sino un "yo puedo"”.

Es cierto, añade Henry, que la comprensión del cogito como un "yo puedo", como acción y como movimiento más bien que como un "yo pienso", no es exclusiva de Biran, sino que puede ser encontrada, y el mismo Biran lo ha reconocido, en Schelling, Fichte, Cabanis, Destutt de Tracy, entre otros. Lo decisivo en Biran es que, a diferencia de todos ellos, va a comprender este "Yo puedo", este movimiento y esta acción

como conocimiento interior, como un cogito inmanente: "La consecuencia de esta tesis es infinita, escribe Henry: al afirmar la pertenencia del ser del movimiento a la esfera de inmanencia absoluta de la subjetividad, Maine de Biran nos propone una teoría enteramente nueva del modo de conocimiento por el cual el movimiento nos es dado"10

como movimiento subjetivo.

Por el contrario, en Descartes,

"la consciencia es un yo pienso y todas las modificaciones de la vida de la consciencia no son más que determinaciones del pensamiento, es decir, ideas": en el cogito cartesiano no se trata pues, de deseo, acción o

\footnotetext{
${ }^{9} \mathrm{PhC}, 72-73$.
}

${ }^{10} \mathrm{PhC}, 74$. 
movimiento, sino de las ideas de deseo, acción y movimiento. Descartes es incapaz de pensar algo como el carácter subjetivo del movimiento, esto es, el movimiento subjetivo: "lo que subsiste del movimiento en el seno del cogito cartesiano es únicamente, (...), la idea de movimiento. El movimiento real se efectúa en otra parte, en la extensión. El movimiento subjetivo no es pues mas que el dibujo interior y por sí mismo ineficaz de ese movimiento real"11.

Pero, ¿dónde ocurre este movimiento real? Respuesta: "el lugar donde se cumple el movimiento real es el cuerpo, lo que significa que en el cartesianismo (...) el movimiento pertenece al ser transcendente ${ }^{12}$ del que él es una determinación"13.

Maine de Biran, piensa Henry, contrariamente a Descartes, fue capaz de dar cabida a los correlatos reales de estas ideas en el seno mismo de la subjetividad, es decir de la conciencia y esto, en virtud de la ruptura que opera con el monismo ontológico ${ }^{14}$ cartesiano que sólo permitía a la intuición abrir el ámbito del ser.

Así es como el cuerpo, expulsado por Descartes de la esfera de la subjetividad y más bien considerado como un intermediario entre el alma y sus movimientos sobre la extensión, va a entrar en la definición del Yo biraniano, comprendido ahora como un cuerpo transcendental idéntico al conjunto de poderes que tiene sobre el mundo. En esta identificación impensable por el cartesianismo, entre el ego, el esfuerzo, el movimiento y el cuerpo subjetivo radica, afirma Henry, la posibilidad para el ego biraniano de actuar "directamente sobre el mundo".

$11 \mathrm{PhC}, 78$.

12 Pues el cuerpo es pensado por Descartes como un elemento del ser transcendente.

${ }^{13}$ Ibid.

${ }^{14} \mathrm{El}$ cartesianismo se presenta en esta primera lectura de Henry como un falso dualismo ontológico: si bien reconoce dos substancias heterogéneas, ambas llegan al ser por obra de un mismo modo de aparecer, la intuición. En efecto, si ser es aparecer, estamos ante un monismo ontológico. L'essence de la manifestation se presenta precisamente como una critica radical del "monismo ontológico" que, desde Grecia habría dominado la casi sin excepciones, el pensamiento filosófico occidental. 


\section{La desvalorización de la afectividad}

Michel Henry sostiene en la Phénoménologie du Corps que Descartes ha otorgado un privilegio de orden axiológico al conocimiento teórico e intelectual "que es como una aprehensión impasible del ser matemático y en el que no nos es puesto ni por los sentimientos ni por las pasiones." Precisamente en esta suerte de impasibilidad propia del conocimiento matemático se apoya el privilegio exorbitante que generalmente se le ha otorgado o, dicho de otro modo, "la idea propia de todo intelectualismo, a saber, que la afectividad es algo inferior $y$ que no puede pertenecer a la pura esencia del pensamiento".

Es, justamente, la tonalidad afectiva propia del pensamiento matemático lo que explica su prevalencia por sobre, la experiencia estética por ejemplo a la hora de asentar la validez universal que requiere el saber científico. Descartes no logra nunca comprender que esta claridad y estabilidad propias del saber matemático constituyen la prueba misma del carácter afectivo del pensamiento y no duda en devaluar la afectividad.

"La filosofia de Descartes -escribe Henry- establece entre los varios Erlebnisse una jerarquía que no puede descansar más que sobre consideraciones (...) de orden axiológico. La conceptualidad matemática es considerada superior a la pasión, a pesar de la identidad de su estatuto fenomenológico. La esencia del pensamiento, es por lo tanto identificada con el pensamiento de tipo matemático, con el conocimiento intelectual $\mathrm{y}$ teórico puesto que cada pensamiento afectivo es inferior y en cierto modo, heterogéneo con el pensamiento puro ¿Cuál es el origen de esta desvalorización de la afectividad o de esta sobrevaloración del puro conocimiento teórico? Este origen reside en la tonalidad afectiva del conocimiento intelectual, en el pathos particular de su certeza propia"15.

Podemos comprender entonces que, en rigor, no se ha despreciado la vida afectiva en general, sino solamente algunos de sus modos "mientras que otros, aquellos que vivimos en nuestra vida teorética, fueron revestidos de un valor positivo por razones que son precisamente de orden afectivo y que residen en el contenido afectivo particular de estas experiencias"16.

\footnotetext{
$15 \mathrm{PhC}, 198$

$16 \mathrm{PhC}, 200$ ss.
} 
El Descartes que Henry nos hace descubrir en la Genealogía del Psicoanálisis (1985) y que abordaremos en la continuación inmediata de esta intervención ha comprendido, por el contrario, que la afectividad no es una determinación exterior al pensamiento sino su posibilidad más interior: su esencia. Dado que todo erlebnis posee una naturaleza afectiva entonces, escribe Henry,

"no hay motivo para distinguir algunos Erlebnisse afectivos y otros que no lo fueran. Todas nuestras experiencias, en tanto son diferentes modalidades del vivir, llevan en sí lo que es precisamente el carácter originario de cada vida y de cada experiencia, y que nosotros llamamos aquí, a falta de algo mejor, una tonalidad afectiva. La impasibilidad propia del conocimiento teórico no es mas que una determinación entre otras de esta tonalidad, una determinación quizá mas sutil, en su carácter aparentemente privativo, pero no privado de lo que hace la esencia de la afectividad".

\section{Descartes II: una filosofía del Comienzo}

El halo de no apariencia que rodea lo aparente y rehúsa ofrecerse a la intuición ha hecho fracasar el proyecto cartesiano. No solo el intuicionismo condena al pensamiento a la efectuación, por principio infinita, de verdades particulares, sino que además el perfecto llenado de cada una de las intencionalidades cognoscitivas no es posible: ni siquiera esta colección de verdades ha de cumplir con el ideal de la adecuación perfecta. Más aun, si la intuición es el aparecer exclusivo por el que todo dato emerge de la nada y se mantiene en el ser, surge inevitablemente la cuestión -decisiva- de saber por qué medio conquista su realidad el propio acto intuitivo. En el intuicionismo el aparecer del aparecer finalmente resulta incomprensible ${ }^{17} \mathrm{y} \mathrm{la}$ filosofía del absoluto comienzo debe entonces renunciar a su pretensión de absolutidad.

$17 \mathrm{El}$ complejo análisis de Henry en EM refiere la intuición a su esencia, "la Transcendencia" para mostrar que el aparecer ekstático -como lo supieron ver Kant y luego Heidegger- tiene lugar sobre fondo de un horizonte proyectado por la misma transcendencia. Queda entonces en evidencia, al tratarse la cuestión del aparecer del aparecer, que la Transcendencia no puede ella misma -más que al precio de un absurdo filosófico que es el del monismo- adquirir su propia realidad sobre el fondo que ella misma debiera previamente proyectar. 
El Descartes que nos descubre la Genealogie de la Psychanalyse de 1985 lo ha comprendido antes que nadie. En este libro, Henry propone una notablemente original ${ }^{18}$ repetición filosófica del cogito que ocupa, junto con su comentario, dos de los capítulos centrales de la obra.

\section{El sentido del Comienzo cartesiano}

El proyecto cartesiano, escribe el Henry, es el de una filosofía radical y primera. Su primer objetivo es la búsqueda del Comienzo y no la de un Método para alcanzar el Comienzo. Y es que "ninguna búsqueda de método sería posible si no dispusiese ya de un punto de partida asegurado... en el mismo comienzo"19. Sin embargo, ¿las Meditaciones que buscan el Comienzo no obedecen a la primacía del método?, ¿no se despliegan según un orden de razones que busca la puesta en evidencia? Sin ninguna duda. Pero, reflexiona Henry cacaso aquello de lo que las Meditaciones hablan, el Comienzo, está constituido por un tal orden y por evidencias? En absoluto: lo en cuestión, el Comienzo, se revelará a Descartes, afirma Henry, precisamente poniendo fuera de juego toda evidencia así como también el medio de luz donde la evidencia es posible.

Esta habría sido la genial intuición de Descartes: afirmar la pertenencia de su propio procedimiento a aquello que ya se produce y que lo precede de algún modo haciéndolo así posible al mismo tiempo que hace posible a toda otra cosa ${ }^{20}$. Afirmar pues el carácter dependiente y segundo que el pensamiento posee respecto del Comienzo.

No confundamos nos advierte Henry: el cartesianismo es el comienzo de la filosofía moderna pero no es el Comienzo: la filosofía moderna, toda filosofía, suponen muchas cosas que las preceden. El Comienzo no es lo nuevo, la filosofía cartesiana, el orden de sus razones sino, escribe Henry, lo antiguo y lo mas viejo. Y la filosofía cartesiana tiene la lucidez de dirigirse

${ }^{18}$ No discutiremos aquí la validez de esta potente lectura henryana de Descartes. Nos parece, en todo caso, que al menos el momento "henryano" de Descartes es indudable, como lo atesta sin hacer violencia a la letra cartesiana la "nube de textos" que nos presenta Henry. Es esto suficiente, a nuestro entender, para provocar la exigencia de una nueva inteligibilidad sobre la totalidad de la obra de Descartes.

${ }^{19} \mathrm{GP}, 17$.

${ }^{20} \mathrm{Ibid}$. 
concientemente a lo más antiguo para hacer pie y poder comenzar ${ }^{21}$. Se trata, añade Henry, de un Comienzo "que comienza y no deja de comenzar". ¿Y qué es lo que comienza en un sentido radical? el ser, responde Henry, si es cierto que nada sería si el ser no hubiese ya desplegado su propia esencia para recibir en ella todo lo que es ${ }^{22}$.

\section{E1 Comienzo: el aparecer del aparecer como conciencia}

Hay en Descartes, algo como la idea de una ontología fenomenológica ${ }^{23}$. En efecto, en lo que a la filosofía de Descartes concierne, Henry revisa ahora y desanda en parte el camino recorrido en $L$ 'essence de la Manifestation y en Phénoménologie du corps.

la cuestión de la esencia del aparecer lo que nos conduce al corazón del cartesianismo y no la búsqueda de una realidad óntica privilegiada que satisfaga la sed de evidencia y de apodicticidad que anima a la razón. Los textos fundamentales de Descartes siempre intentan develar la posibilidad más última de la esencia del aparecer y, en lo que atañe al problema del Comienzo, la meditación cartesiana es absolutamente ajena a una problemática que gire en torno de la evidencia y la intuición.

A la esencia comenzante y primera Descartes la comprenderá como el aparecerse a si del aparecer y la llamará "cogito", conciencia" o pensée. Un cogito que remite de este modo a una interioridad absoluta ${ }^{24}$. Nada tiene que ver, en todo caso, con el cogito que munido del Método se encamina hacia ella, es decir, con lo que habitualmente entendemos por "pensar".

La genialidad de Descartes, según Henry, reside en haber concebido que en el comienzo no se halla un ente, ni siquiera su aparecer, sino el aparecer del aparecer, la autofenomenalización de la pura fenomenalidad. Y esto significa que el Comienzo es absoluto y plenamente fenomenológico. El primer aparecer no está ligado al ente sino, en una interioridad y una autonomía que

\footnotetext{
21 Ibid., 18.

22 Ibid.

23 GP, 17.

24 GP, 32.
} 
la filosofía casi nunca pudo concebir ${ }^{25}$, a su propio aparecer. el comienzo no es cogitocogitatum sino cogito.

La autonomía del cogito respecto de todo ente, que Henry atribuye a Descartes, significa la autarquía del aparecer original: en efecto, argumenta Henry, "¿qué es lo que ya se encuentra allí antes que cualquier cosa que aparece y cuando aparece si no es el aparecer mismo y puro como tal?" 26 .

Sólo el aparecer constituye lo verdaderamente inicial del comienzo, pero no en tanto modela el aparecer de la cosa y su venida al ser -tal comienzo sólo seria el del ente- sino en un sentido aun más originario: en el Comienzo, el aparecer es lo que aparece $y$ también a quien le aparece: "solo en esta medida, escribe Henry, el aparecer es idéntico al ser y lo funda", "en tanto fulguración luminosa que es iluminación de ella misma y no de otra cosa, en tanto aparecer del aparecer que expulsa la nada y toma su lugar"27.

\section{La "contrarreducción" cartesiana que libera el Comienzo: una crítica del intuicionismo}

El procedimiento cartesiano que busca el Comienzo es una reducción. En rigor, y este habría sido el mayor problema para su comprensión, no hay, afirma Henry, una única reducción en Descartes sino dos reducciones que persiguen objetivos diferentes y que terminan por oponerse radicalmente.

La primera reducción, es en todo análoga a la reducción galileana, es decir a la ciencia moderna, que por acto de imaginación transforma las propiedades sensibles en una naturaleza estable constituida por formas geométricas.

Descartes habría tenido, según Henry, la excepcional inteligencia de retomar y asumir la reducción galileana que abre al conocimiento matemático de la naturaleza pero al mismo tiempo operar una contra-reducción,

25 Henry reconoce en Maister Eckhart, Descartes, Maine de Biran, Marx y finalmente, en sus últimos trabajos, en el primer cristianismo, la línea histórica que recoge esta tesis filosófica inmanentista.

${ }^{26} \mathrm{GP}, 18$.

27 Ibid. 
una "reducción fenomenológica radical" que "va a tematizar aquello que la reducción galileana puso fuera de juego y lo va a pensar hasta su límite"28.

La mayor dificultad que presenta la lectura de los grandes textos de Descartes es, de acuerdo con Henry, que ambas reducciones, que entre sí están en conflicto, se entrecruzan y se recubren constantemente en función de los diversos objetivos perseguidos.

La Segunda Meditación sería el más claro ejemplo de este recubrimiento que lleva tanto a producir la impresión de identidad entre ambas reducciones como a la evidencia de su oposición. La Duda, sostiene Henry, lamentablemente designa los dos procesos de reducción. Cuando la duda es duda del mundo sensible, se trata de una reducción de tipo galileano. Esta reducción, tras dejar de lado los sentidos y la imaginación por considerarlos engañadores, desemboca en el verdadero poder de conocimiento y nos ofrece la primera definición del pensamiento: "mens, sive animus sive intellectus, sive ratio". Esto es, el ver puro transcendental que nos permite acceder al conocimiento de los cuerpos, al conocimiento verdadero de la naturaleza. La reducción es en este caso metafisica. El célebre análisis del trozo de cera y de los hombres con sombrero del final de la Meditación Segunda, refiere al conocimiento de la res extensa y no es más que una "repetición", escribe Henry, en los mismos términos, del análisis galileano del Saggiatore. Esta primera reducción, que revela la esencia del conocimiento de los cuerpos nada tiene que ver con la problemática que apunta a develar el Comienzo, es decir, con la problemática del conocimiento del alma. Se trata, por el contrario, de la fundación del racionalismo transcendental, la fundación de una "teoría transcendental del conocimiento del Mundo".

Muy distinta es la "contrarreducción" que opera Descartes, su "reducción fenomenológica radical": en vez de dejar de lado los sentidos y la imaginación, los conserva y afirma su carácter de indudables $y$ de absolutos. En esta contrarreducción, sostiene Henry, se origina la segunda definición del

${ }^{28}$ Henry, M; "Descartes et la question de la technique", en N. Grimaldi y J.-L. Marion (editores.), Le Discours et sa méthode, PUF, Paris, 1988, p. 285-301. En adelante $(D T)$. 
pensamiento - que se opone a la primera- pues esta vez enumera en primer lugar, como perteneciendo a la esencia misma del cogito, el sentir y la imaginación ${ }^{29}$.

Pero ¿cómo pueden el sentir y la imaginación, pregunta Henry, ser eliminados por una reducción para permitirnos reconocer la esencia del cogito y subsistir bajo la otra? La respuesta más rápida sería: son eliminados en tanto facultades empíricas -como consecuencia de la desaparición del cuerpo que las sostiene- y permanecen en tanto facultades transcendentales consideradas por ellas mismas, y a este título, en tanto datos irrecusables. Hay, empero, segun Henry, una razón más profunda: la reducción fenomenológica radical no sólo retoma y conserva aquello que la reducción galileana había dejado fuera de juego sino que pone fuera de juego al proyecto galileano mismo: con ella, el principio del conocimiento verdadero ya no reside en el intellectus ni en la ratio. "La duda contra las verdades racionales, escribe Henry, es una duda contra toda verdad posible, una duda contra el intellectus y contra la ratio que ya no son más fiables".

Para posibilitar esta duda ciertamente fue precisa la intervención de alguna instancia capaz de quebrar la ratio y el intellectus y de fingir al menos durante un momento que son potencia de error. Se piensa en la voluntad infinita, capaz de superar al entendimiento y seguir a ciegas durante un momento el proyecto que la ratio ya no puede llevar sobre sus hombros. Pero Henry nos recuerda que la significación de lo que denomina la "contrarreducción" de Descartes es fenomenologica. Dicho de otro modo: la crítica cartesiana de la ratio no es simplemente especulativa sino fenomenológica. En ese caso, lo que debiéramos intentar comprender ante todo, sugiere Henry, es qué es la ratio y cuál es el motivo fenomenológico de su falibilidad y no quién la vulnera.

${ }^{29}$ Hay en la Segunda Meditación dos definiciones de "pensée". Una por su esencia: "mens, sive animus, sive intellectus". Según Henry no es apta para dar cuenta del Comienzo y refiere más bien al cogito comprendido como un conocimiento del cuerpo. La segunda definición del cogito, esta vez por enumeración de sus modos, da cuenta de su comunidad substancial en el videor. "Qué es una cosa que piensa? Una cosa que duda, que concibe, que afirma, que niega, que quiere, que no quiere, que imagina tambien y que siente". Y es que "sentir" es "pensar que se siente", imaginar es "pensar que se imagina", etc. 
La Regula I, nos recuerda Henry, define fenomenológicamente la Ratio como una luz transcendental, "Sapientia universalis scientid", bona mens, naturale regionis lumen". Henry transcribe el texto de Descartes: "una luz que permanece siempre una y la misma por diferentes que fueran los objetos a los que ella se aplica y que no recibe más cambios de estos objetos que la luz del sol de la variedad de cosas que ella ilumind".

La duda contra la ratio es entonces duda cartesiana contra esta luz, luz ahora dudosa, que siendo indiferente a las verdades racionales que ella misma ilumina, las vuelve entonces dudosas junto con ella.

La duda contra la ratio es duda contra el ver de la ratio, y Michel Henry anota:

"es porque el ver de la ratio, el intueri del intellectus, la inspectio mentis ve todo aquello que ve en ese horizonte transcendental de visibilidad que es la luz natural (más profundamente, porque esta luz no es un poder de visibilidad, un hacer ver, una luz, sino, por el contrario, una potencia de deformación, un inducir en error) que todo lo que ve y puede ser visto en esta supuesta luz que es el mundo, ya sea un contenido sensible o intelectual, es falso o dudoso".

Se precisa entonces el sentido y el alcance de la reducción cartesiana: lo reducido y desechado conjuntamente con la totalidad del ente es el borizonte de visibilidad desplegado por el ver, por el intueri.

$\mathrm{Y}$ así, falso es el ver en la luz, falsa es la evidencia de todo cuanto en ella aparece porque la luz sobre la que aparece es una potencia de engaño o de error.

Desde luego que esto no sólo vale para lo visto por los ojos, sino para cualquier visión: por ejemplo para aquella del entendimiento donde 2 más 3 son 5 y donde parece muy seguro que si pienso entonces existo:

"toda visión me induce en error, ya que lo dudoso no proviene en verdad del carácter de cada contenido particular que me ofrece el ver sino de la calidad ontológica del medio que todo ver despliega cada vez que da a ver".

¿Fue la voluntad infinita quien interrumpió el proyecto de la razón y lo puso en sus propias manos para seguir el camino trazado por el método? A esta pregunta responde ahora Henry: 
“¿pero cómo podríamos saber, bajo este derrumbe de toda facultad de conocimiento que ha sido la voluntad la que ha puesto en cuestión la visibilidad, cuál es su naturaleza o incluso si esta voluntad existe?" "Ya no se trata en Descartes de exhibir un fenómeno privilegiado que escape a la perversión de todos los otros bajo una luz evidente, pues esta luz ha sido descalificada".

Se revela entonces aquí, la radicalidad asombrosa del proyecto cartesiano: éste pretende reconocer y dar un estatuto filosófico a un modo de aparecer cuya obra no dependa en nada de la luz.

Animado por su fidelidad al Comienzo, habiendo abandonado al término de la duda el ente en su totalidad y la luz sobre la que éste es dado, esto es, el mundo, Descartes va a operar ahora una distinción decisiva en el plano ontológico mismo, entre dos modos de aparecer. Afirmará, al mismo tiempo, con obstinación la primacía de uno de estos modos sobre el otro.

¿Cuáles son estos dos modos? Estos dos modos de aparecer son, ante todo, dos modos de conocimiento. El primero de ellos es aquello que Descartes llama "conocimiento del cuerpo": es el ver, ya sea el de los ojos o incluso el ver puro y transcendental que queda tras la reducción. Un conocimiento, un ver cuya estructura ekstática lo determina como cogitocogitatum : el ver de lo visto en la luz. Un ver que ha resultado ser por esencia incapaz de garantizar la certeza del pensar. Esto es al menos lo que nos ha mostrado el análisis henryano de su bancarrota en la primera Meditación. Pero, y esto es aun más importante, un ver que es incapaz de garantizar su propia realidad.

$\mathrm{Y}$ es que este modo de aparecer que pone a distancia lo conocido, este intueri, este conocimiento ekstático, este ver empírico o reducido -aquí la diferencia es inesencial- siempre presupone su propio aparecerse a sí, es decir, supone un poder de manifestación que le permita llegar a sí, apropiarse de sí interiormente para ser un ver $y$, ante todo, para ser.

Este es, afirma Henry, el segundo modo del aparecer, la autorrevelación del éxtasis en la inmanencia ${ }^{30}$. Si el ver es el aparecer del Mundo, el Comienzo es el aparecer del aparecer.

${ }^{30} \mathrm{GP}, 34$. 
Esta es la tesis que gobierna la nueva lectura henryana de Descartes, en quien encuentra el precedente de su propio pensamiento: el aparecer del aparecer posee efectividad fenomenológica y autonomía absoluta, $y$ en él consiste el ser ${ }^{31}$. En efecto,

“Cómo podría el ser -escribe Henry- siquiera una fracción del ser, quedar separado del aparecer? De suceder esto, sería el ente y no el ser. ¿No es el ente el que reclama su aparecer a un poder que no le pertenece y que siempre encuentra fuera de sí? En el alba de la filosofía moderna, cuando con Descartes la esencia de la Psyché es definida por primera vez, no hay ninguna escisión entre el mostrarse del ser y algo que en él habría de escapar a la fenomenalidad: algo semejante arruinaría toda esta problemática"32.

\section{Videre videor ("Me parece que veo")}

¿De qué medio disponemos para reconocer la realidad y la obra de este supuesto aparecer sui generis si la luz transcendental de la ratio, si toda luz, al término de la duda, se ha apagado? ¿No estaremos, con la afirmación henryano-cartesiana de un aparecer inekstático, ante una declaración puramente especulativa?

La reducción operada en la Primera Meditación ha eliminado, junto con el poder de la ratio, la res extensa. Es en ese contexto, o más exactamente, en esa ausencia total de contexto, que interviene en la Segunda Meditación la declaración cartesiana "at certe videre videor, audire, calescere", ("al menos es cierto que me parece que veo, oigo, siento el calon»). Esta declaración es decididamente asombrosa pues quien tiene aqui la semblanza cierta de ver carece, en virtud de la reducción, de ojos para bacerlo y por otra parte, no babiendo mundo, no bay nada para ver, quien tiene la cierta semblanza de sentir calor carece de cuerpo y por otra parte nada exterior podría venir a provocarlo, etc.

31 Ibid., 18.

${ }^{32}$ Ibid., 22. 
Esta declaración pronunciada en la Segunda Meditación en régimen de reducción radical, ofrece al cogito cartesiano, afirma Henry, su formulación mas radicaß ${ }^{33}$.

Este acósmico "me parece muy cierto que veo", es, en todo caso, el último resto de la reducción y ha de llevar en sí indiscutiblemente el aparecer incontestable, el Comienzo.

La declaración cartesiana se vuelve perfectamente transparente, aun en régimen de reducción radical, si se comprende que la semblanza por la que me parece que veo nada tiene que ver con el aparecer en que se despliega el ver para alcanzar lo visto en una evidencia, es decir, con la engañosa luz transcendental. Este es el núcleo de la segunda lectura henryana de Descartes.

La fórmula "videre videor" que interviene en la Segunda Meditación expresa, según Henry, la descomposición del pensamiento según los dos modos fundamentales en que se fenomenaliza la fenomenalidad 34 : la autoafección inmanente (videor) y el ekstasis (videre). En efecto, Henry concibe el cogito cartesiano como una reducción fenomenológica que separa el ver ekstático que avanza en la luz, que se representa y que "ve", y la pura experiencia interna de sí de este ver, en su afectividad nocturna, en su inmediatez absoluta, en su ignorancia de cualquier "enfrente", de cualquier "ver" y de todo lo "visto".

Ha subsistido a la destitución cartesiana del mundo la experiencia interna de un ser que ya no tiene ojos ni oídos ni cuerpo y que, incluso, tal vez no existe ${ }^{35}$. Ha subsistido una experiencia interna absolutamente segura que para su efectuación no le debe nada al mundo. Esto significa

${ }^{33}$ GP, 24. A diferencia del cogito "herido" de Ricoeur o del cogito levinassiano abierto por la idea de infinito-ambos sensibles al movimiento cartesiano de la Tercera Meditación y críticos, al igual que Heidegger, de la idea de plenitud como paradigma ontológico o metafísico, el cogito "henryano" encontrará lo esencial en la Segunda Meditación: esto es, la posibilidad de pensar la plenitud fuera de los supuestos ontológicos griegos que, exclusivamente atentos a dar cuenta del ekstático aparecer del mundo habrían sido ciegos a la estructura de inmanencia en que se efectiviza la Vida como pathos. A diferencia pues, de Heidegger, Ricoeur y Levinas, Henry sostiene que la plenitud nunca fue correctamente pensada. Este segundo Descartes de M. Henry interviene precisamente como primer pensamiento de una plenitud no griega. 34 GP, 52.

${ }^{35} \mathrm{GP}, 25$. 
fenomenológicamente que la interioridad del cogito, esto es, el videor, no se efectiviza relativamente a una "exterioridad" y como su negación sino que se manifiesta positivamente como "interioridad" en virtud de su propio modo de aparecer: el videor es la experiencia misma de la inmediatez. Es en este sentido un cogito sin cogitatum.

Por este motivo, explica Henry, es que la imaginación y los sentidos caían en una reducción mientras que resisitían en la otra: caían en tanto modalidades del ver, en tanto se arrojan en la luz. Y con razón pues todo lo visto por ellos, podría finalmente ser un sueño sobre fondo de luz natural. No se derrumban en cambio ante la reducción, en tanto la experiencia interna en que consisten, es lo que es, un pathos, que se teje fuera de la luz, independientemente de la existencia o de la inexistencia de un mundo o de un Dios transcendente. Resisten pues, en tanto su esencia es también un videor.

Para el Descartes del comienzo, afirma Henry, existir es sinónimo de aparecer, de manifestarse: videor significa precisamente esto, la semblanza primitiva, la capacidad original del aparecer, independientemente de la credibilidad que otorguemos a lo visto por la visión.

"Videor" es, en suma, "el aparecer en virtud del cual el videre se aparece ante todo a sí mismo"36. Sin embargo, la semblanza por la que el videre se alcanza interiormente a sí mismo cada vez que ve, la certeza inmanente de su plenitud, no es el mismo tipo de semblanza que ofrece el videre cuando nos da a ver lo visto, conformemente a los diversos grados de evidencia.

"Videre videor", nos advierte entonces Henry, no significa una simple duplicación del videre intencional, duplicación "por la cual, en régimen de epojè, habría de nacer una certeza, la primera". Semejante duplicación sería perfectamente incapaz de ofrecernos una certeza pues lo que el videre no puede ofrecer a priori en virtud de su propia naturaleza - una certezatampoco lo podría ofrecer tomándose a sí mismo por objeto. Precisamente por ese motivo había sido desechado por el cogito como medio apto para alcanzar alguna verdad.

"Videre videor" no es pues "ver que", o "pensar que", o "considerar que" o, "tener por verdadero que", sino, afirma Henry, aquello que el

${ }^{36} \mathrm{GP}, 27$. 
Descartes del comienzo entiende por "pensar": saber, el sentirse inekstático en que todo ver se alcanza interior e inmediatamente y asi, puede ser lo que es y ver lo que ve.

La continuacion inmediata del texto de Descartes en que interviene el «videre videor» pareciera ofrecer un fortísimo sustento a esta lectura henryana: «al menos es cierto que me parece que veo, oigo, siento el calor; esto no puede ser falso y esto es, propiamente, lo que en mi se llama sentir, y esto precisamente, es pensan ${ }^{37}$.

$\mathrm{El}$ cogito cartesiano se dice entonces: "siento que pienso, soy38". En lenguaje cartesiano: "cuando veo, o, cosa que no distinguiré mas, cuando pienso ver", donde pensar es el videor del videre, el sentir inmanente que construye internamente al videre y le ofrece su única realidad ${ }^{39}$.

Videre y videor son en definitiva, dos modos de manifestación, dos "cómos" del aparecer que "difieren en la materialidad de su aparecer puro" 40 . En el primer modo, en el videre y en su luz, lo visto siempre es exterior a la luz que con indiferencia lo rodea y lo mantiene en la presencia. Aquí lo que aparece es exterior a su propio ser: un ser que le es garantizado sólo un instante por esta luz. Esta condición ontológicamente precaria del fenómeno ante el videre ekstático es su objetividad. Por eso es que en la luz el fenómeno objetivo se manifiesta siempre con un aire de contingencia y ofrece el aspecto de simple fachada de un ser sin interior. Por esta contingencia que resulta de la

${ }^{37}$ Descartes, Meditaciones Metafisicas, Meditación Segunda.

38 Ibid. Notemos que, además de la presencia del "siento", la coma reemplaza en esta formula de Henry al ergo del Discurso del Método: ninguna inferencia puede llevarme del pienso al soy dado que la ratio ha sido destituida en la Primera Meditación. El pienso es aquí la realidad misma del soy, su autoafección inmanente. Dicho de otro modo, habría un Descartes propiamente fenomenológico en esta Segunda Meditación que no intenta "demostrar" nada sino, más bien, mostrar o dejar que el comienzo se muestre.

39 "Sentimus nos videre", escribe en carta a Plempius (citado por Henry, GP), para distinguir el ver humano -un ver verdadero pues portador de un videor- del supuesto "ver" animal, un videre sin videor, es decir, tan imposible como la visión de una cámara que enfoca el mar sin que nadie mire a través de ella. Un videre carente de "esta especie de conocimiento interior que siempre precede al adquirido", Respuestas a las Séptimas objeciones.(citado por Henry, GP, 32).

${ }^{40} \mathrm{GP}, 36$. 
exterioridad entre lo dado y el poder que lo da, por esta fragilidad ontológica del objeto, es que la res extensa ha sido revocada en duda.

Por el contrario, en el modo originario de revelación del ser, en el videor, todo lo que aparece se muestra tal como es, en su realidad, iluminado por su propia luz, luz iluminada por sí misma, en una materialidad que sólo es impensable porque su "qué", que es también su "como", no se prodiga como una luz sino más bien como un fuego: la autoafección inmanente en que consiste la vida, su esencia, su afectividad, el becho puro y simple de sentirse a si mismo, y no una cosa que se siente a si misma, la subjetividad.

\section{Descartes y la fenomenología}

$\mathrm{Al}$ término de la reducción cartesiana ha subsistido la visión a título de fenómeno puro; sin embargo ¿no es también indudable, pese a toda esta argumentación henryana, la efectividad de la luz transcendental y en ella la de todo lo que ella ilumina - a condición de que lo tomemos tal como se muestra $y$ respetando esta mostración?

Henry sostiene que es precisamente en la respuesta a este interrogante que se separa el proyecto cartesiano y el de la "fenomenología histórica" 41 que al no comprenderlo, lo ha pervertido ${ }^{42}$.

Para Husserl, "cogito" es "ego cogito cogitatum de modo tal que cogitatio es la intencionalidad, el bacer ver en el que es visto todo lo que puede ser visto, de modo tal que el ser visto es el cogitatum qua cogitatum. En Heidegger la situación es aun más grave, cogito es yo me re-presento, pongo ante mi, Vor-stellen. Es cierto que incluso en Descartes podríamos encontrar ciertas problemáticas cuyo punto de partida es el cogitatum, la realidad objetiva de la idea en la Tercera Meditación, por ejemplo"43. El Método mismo nos invita a partir del cogitatum: "no tomar nunca una cosa por verdadera que yo no conozca evidentemente como tal".

Sin embargo, la afirmación de que el cogitatum qua cogitatum es lo incontestable, sostiene Henry, ha perdido lo esencial del pensamiento de

41 "Fenomenología histórica" es la denominación henryana para la fenomenología intencional, por oposición a su "Fenomenología Material".

${ }^{42} \mathrm{DT}$.

${ }^{43}$ Ibid. 
Descartes. Pues su pensamiento no consiste en fundarse sobre la evidencia y tenerla por principio del conocimiento, más aun, en tenerla por principio de los principios, sino más bien en legitimarla. Una legitimación que consiste para Descartes en mostrar que todo ver sólo puede ver sobre fondo de un no ver, sobre fondo de una semblanza absolutamente distinta a aquella por la que el ver alcanza lo que ve.

Finalmente, esta semblanza es exactamente lo que nosotros somos: en términos cartesianos, un puro "conocimiento del alma", una idea sin realidad objetiva ${ }^{44}$, un cogito sin cogitatum 0 , como lo escribe Henry:

"la subjetividad absoluta entrevista por Descartes que marca una total ruptura con la fenomenalidad griega, con el mundo griego donde fenómeno significa lo que brilla en la luz, el ekstasis del mundo". "En la Noche abismal de su interioridad radical -añade el filósofo- donde no hay ni ekstasis ni luz ni mirada, la subjetividad absoluta hace la experiencia de sí, ella es la Vida. De tal manera que la Vida sólo es posible alli donde una tal subjetividad ha desplegado su esencia, como aquello que es la experiencia de sí y no de otra cosa, de una realidad objetiva o de una alteridad".

El Artículo 26 del Tratado de las Pasiones del Alma, a menudo citado por Henry en sus exposiciones sobre la filosofía de Descartes, nos ofrece la oportunidad de medir la fuerza de la lectura henryana. En ese artículo, la hipótesis del sueño permite a Descartes operar una reducción que libera una realidad última indiferente a todo ver, y ante todo, al ver de la reducción misma (siempre que se comprenda la reducción como un procedimiento intuitivo). Lo liberado es una realidad puramente afectiva -el sentimientocuya absoluta seguridad ontológica, es decir, cuya realidad, sólo puede explicarse por la estructura de inmediación en que tiene lugar su aparecer: lo que allí aparece es, bajo el modo de una autoafección, el propio aparecer. Esta es la explicitación ontológica de la afectividad, esencia cartesiana de la subjetividad, al menos para el Descartes que busca el Comienzo:

44 "Por Idea entiendo esta forma de cada uno de nuestros pensamientos por la percepción inmediata de la cual tenemos conocimiento de estos mismos pensamientos" (AT VII, 160), citado por Henry. La Idea es, pues, en la interpretación henryana, otro nombre para la subjetividad. 
"Así a menudo, escribe Descartes, cuando se duerme e incluso a veces estando despiertos, imaginamos tan fuertemente ciertas cosas que pensamos verlas ante nosotros o sentirlas en el cuerpo por más que no se encuentren allí de ningún modo. Pero, ya sea que estemos despiertos o soñando, no sería posible sentirnos tristes o emocionados por alguna pasión y que el alma no tenga en ella de un modo verdadero esta pasión". "Es posible equivocarse respecto de las percepciones que se refieren a objetos que están fuera de nosotros (...) pero...no es posible hacerlo respecto de las pasiones, en tanto están tan próximas son tan interiores a nuestra alma que es imposible que esta las sienta sin que sean verdaderamente tal como ella las siente".

Henry sostiene que desde el final de la Segunda Meditación Descartes habría "retrocedido" ante el descubrimiento de esta subjetividad ontológica, puramente pática y acósmica. No habría sabido mantener su pensamiento en el vértice de tensión requerido por un pensamiento de la inmanencia. $\mathrm{La}$ intervención de un Dios transcendente en la Tercera Meditación ${ }^{45}$, la ambigüedad que, en adelante, habrá de pesar sobre la concepción de las pasiones ${ }^{46}$, y en general, sobre todos sus conceptos, se explicarían por una involuntaria regresión al aparecer del mundo tomado nuevamente por Descartes como modo exclusivo de fenomenalización de la fenomenalidad.

El Descartes de l'Essence de la manifestation y de la Phénoménologie du Corps, que hemos comentado al principio de este trabajo, habrá constituido, en

45 En sus últimos trabajos, Henry reconoce en lo que denomina el "cogito cristiano de Ireneo" (Encarnacion, \$25) una reducción aun más radical que la operada por el cogito cartesiano: Ireneo, al afirmar que la carne es dada en la Vida, babría sabido reconocer, contrariamente a Descartes, la inmanencia de Dios-identificado con la Vida- a la experiencia de si de la carne.

${ }^{46} \mathrm{La}$ pasión, en efecto, será en general pensada por Descartes, al modo más tradicional, como resultado de la acción del cuerpo sobre el alma. Henry muestra sin embargo cómo esta visión se encuentra en los textos cartesianos constantemente en oscuro litigio con la de la absoluta autonomía y el carácter propiamente ontológico del sentimiento. Por otra parte, afirma filosamente Henry, en el momento en que tiene lugar la identificación del Comienzo con la esencia de la subjetividad y con el pathos, en la Segunda Meditación, no queda justamente ningún cuerpo susceptible de actuar sobre el alma! 
una puesta en perspectiva de la obra total de Michel Henry, el análisis riguroso y fecundo de este Descartes que ya ha perdido el Comienzo.

\section{Resumen}

Con el ego cogito sum, Descartes ha buscado ofrecer un suelo seguro para la edificación del conocimiento científico. Sin embargo, la problemática así instaurada ha olvidado totalmente, como señala Heidegger, elucidar el sentido de ser de ese sum. Puede decirse que toda la obra de Michel Henry constituye una tentativa profundamente original de plantear la cuestión del ego sum sobre un contexto verdaderamente ontológico. Es en esta medida que su meditación sobre la obra y el proyecto cartesiano ocupa un lugar central y permanente. Henry nos descubre en L'essence de la Manifestation (1964) y en Pbénoménologie du Corps un Descartes cuyo proyecto, basado irreflexivamente sobre un procedimiento intuicionista identifica verdad con evidencia y predetermina así su investigación como una imposible tentativa de exhibir el fundamento bajo la luz. Imposible, pues el fundamento, afirma Henry, el ser del ego, es invisible. Esto es lo que ha comprendido el Descartes al que nos abre Henry en su "Généalogie de la Psychanalyse" (1985): el ser del sum no es un "qué" susceptible de ser dado por un ver sino un "como" que se revela sin mediación a sí mismo como patbos. el aparecerse a si de la vida en la inmanencia de su afectividad.

Palabras clave: "Descartes", "afectividad", “intuicionismo", "Michel Henty".

\section{Abstract}

With the cogito ergo sum, Descartes has intended to offer a safe ground for the construction of scientific knowledge. Nevertheless, the problematic thus posed has totally forgotten, as Heidegger points out, to elucidate the sense of the being of this sum. It can be said that the whole work of Michel Henry constitutes a deeply original attempt to raise the question of the ego sum on a truthfully ontological context. Hence, his meditation on Descartes work and project takes up a central and permanent place in his work. Henry discovers to us in L'essence de la Manifestation (1964) and in Pbénoménologie du Corps (1965) a Descartes whose project, based unreflectively on an intuitionist procedure, identifies truth with evidence and thus predetermines his investigation as an impossible attempt to exhibit the grounding under light, which is impossible, given that the grounding, the being of the ego - Henry states-, is invisible. This is what Descartes has understood, according to Henry's "Généalogie de la Psychanalyse" (1985): the being of the sum is not a "that" which may be given by a vision but a "how" that reveals itself without any 
mediation as pathos: the appearing of life to itself in the immanence of its affectivity. Key words: "Descartes", "affectivity", "intuicionism", "Michael Henry". 